\title{
Spectroscopy of the Single-Particle States of a Quantum-Dot Molecule
}

\author{
T. Schmidt, ${ }^{1}$ R. J. Haug, ${ }^{1,3}$ K. v. Klitzing, ${ }^{1}$ A. Förster, ${ }^{2}$ and H. Lüth ${ }^{2}$ \\ ${ }^{1}$ Max-Planck-Institut für Festkörperforschung, Heisenbergstrasse 1, 70569 Stuttgart, Germany \\ ${ }^{2}$ Institut für Schicht- und Ionentechnik, Forschungszentrum Jülich GmbH, Postfach 1913, 52428 Jülich, Germany \\ ${ }^{3}$ Institut für Festkörperphysik, Universität Hannover, Appelstrasse 2, 30167 Hannover, Germany
}

(Received 9 August 1996)

\begin{abstract}
We investigate low-temperature transport through two identical vertically coupled quantum dots in a triple-barrier heterostructure. The current-voltage curve exhibits two series of current steps of different magnitude. On the basis of magnetotunneling measurements, we relate the current steps to the single-particle levels of a quantum-dot molecule. From the absence of many-particle phenomena, an upper limit of $1 \mathrm{~ns}$ is determined for the energy-relaxation time in the double-dot system. [S0031-9007(97)02436-8]
\end{abstract}

PACS numbers: 73.23.Hk, 73.40.Gk, 73.61.Ey

Quantum dots are zero-dimensional systems which exhibit confinement in all three dimensions of space. Rich spectra of discrete single- and many-electron states of such "artificial atoms" were studied by single-electron tunneling (SET) and capacitance spectroscopy [1]. SET through quantum dots, which are coupled via tunneling barriers to two contacts, leads to steps in the currentvoltage curve $I(V)$, whenever discrete dot levels become energetically available for transport [2-4].

Recently, researchers focused on the interplay of two quantum dots in series coupled by a central tunneling barrier [5-14]. The bulk of existing work considers the intradot and interdot Coulomb interaction in double-dot systems (DDS) with many electrons [8-14]. SET between weakly coupled dots was interpreted as a sequential process which requires two discrete levels in the dots to be lined up in energy (taking due account for charging effects). This condition is fulfilled only at certain specific bias voltages which leads to sharp peaks in the $I(V)$ curve $[6,10]$. Experiments on strongly coupled DDS provided evidence for coherent interdot coupling [11,12].

In this Letter, we investigate the single-particle regime of a strongly coupled DDS which was fabricated by imposing a submicron lateral confinement on a triplebarrier heterostructure. The $I(V)$ curve exhibits steps reminiscent of SET in single dots. We attribute these current steps to SET through discrete single-particle states extended over both identical dots due to coherent interdot coupling. Thus, our DDS represents an ionized "artificial hydrogen molecule." From the magnitude of the steps, we estimate the energy-relaxation time in the DDS.

The triple-barrier heterostructure was grown by molecular-beam epitaxy on an $n^{+}$-type GaAs substrate. As shown in Fig. 1(a), the undoped active layers comprise two $6 \mathrm{~nm}$ GaAs quantum wells $A$ and $B$ which are strongly coupled to each other by a $1 \mathrm{~nm}$ thin center AlAs barrier and weakly coupled to two $n$-doped contact layers by $4 \mathrm{~nm}$ thick AlAs barriers. The Si doping profile of the contact layers is graded from $2 \times 10^{18} \mathrm{~cm}^{-3}$ to zero adjacent to the undoped triple-barrier region.
Figure 1(b) shows the conduction-band profile $V(z)$ of the heterostructure in growth direction for zero bias and $V=200 \mathrm{mV}$. It was calculated with a Poisson solver in Thomas-Fermi approximation. From the wafer we fabricated free-standing pillars with Ohmic contacts [2] and measured their dc $I(V)$ curves in a dilution refrigerator at the base temperature of $T=23 \mathrm{mK}$.

In order to characterize the heterostructure, we discuss the $I(V)$ curve of a large-area sample with $d_{p}=$ $2 \mu \mathrm{m}$ pillar diameter plotted in Fig. 2(a). It exhibits two clear peaks due to resonant tunneling through twodimensional subbands which are formed by vertical quantization in the double-quantum well [see Fig. 1(b)]. For zero bias, the lowest levels of the identical quantum
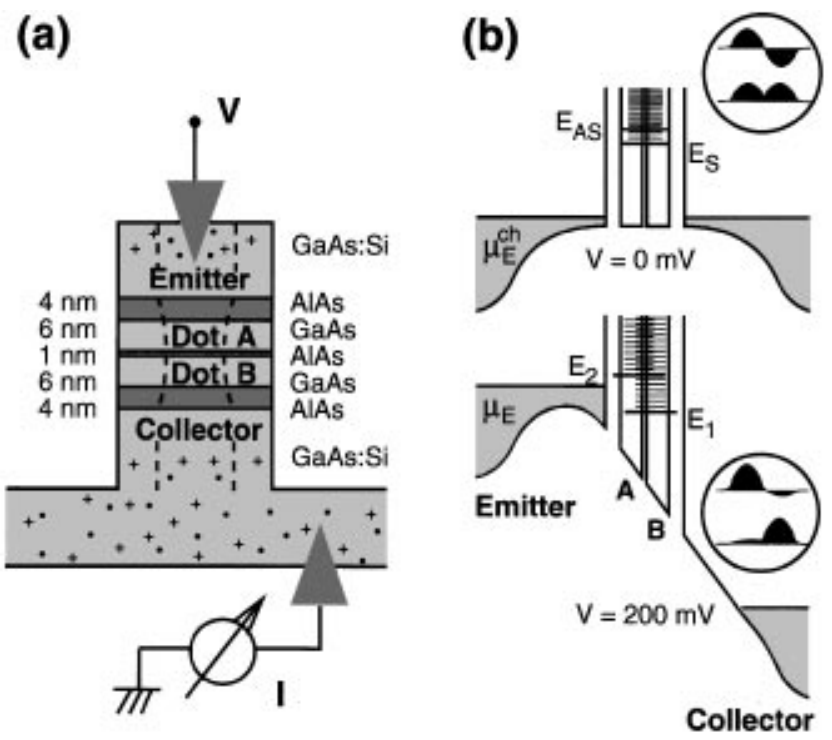

FIG. 1. (a) Sketch of a vertically coupled double-dot device. Dashed lines define the depletion layer at the pillar surface, which confines the electrons to the central core of the pillar. (b) Conduction-band profiles of the heterostructure for $V=0$ and $200 \mathrm{mV}$. Thick lines represent the subband edges of the double-quantum well, thin lines the related discrete double-dot levels. Insets show corresponding wave functions. 


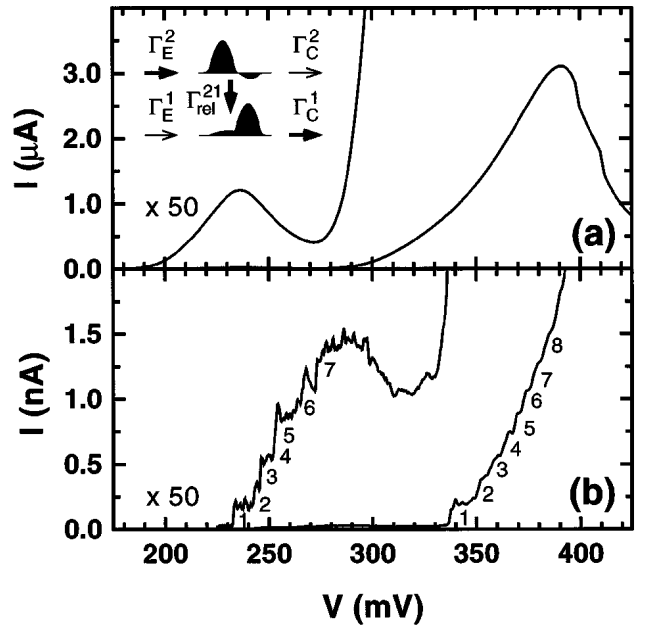

FIG. 2. (a) $I(V)$ curve of a sample with $2 \mu \mathrm{m}$ pillar diameter. The inset illustrates relevant tunneling and relaxation processes. (b) $I(V)$ curve of a device with $250 \mathrm{~nm}$ pillar diameter.

wells split into two levels $E_{S}$ and $E_{A S}$ with symmetric and antisymmetric wave functions, which are completely delocalized over both wells [upper inset of Fig. 1(b).] From the Schrödinger equation, we obtain a symmetricantisymmetric gap of $\Delta_{S A S}=E_{A S}-E_{S} \approx 13 \mathrm{meV}$. In experiment, the current peaks occur at bias voltages of $V>200 \mathrm{mV}$. Under such conditions, the two lowest states are mainly localized in either well $A$ or $B$ [lower inset of Fig. 1(b)], and their calculated energy splitting exceeds the zero-bias gap considerably, $E_{2}-E_{1}>$ $35 \mathrm{meV}>\Delta_{S A S}$. The localization influences the emitterand collector-barrier tunneling rates $\Gamma_{E}$ and $\Gamma_{C}$ since the probability density of the wave functions at one barrier is by almost 2 orders of magnitude higher than at the other one, i.e., $\Gamma_{E}^{1} \ll \Gamma_{C}^{1}$ and $\Gamma_{E}^{2} \gg \Gamma_{C}^{2}$. The low-bias peak in Fig. 2(a) is the consequence of resonant tunneling through subband 1, whereas the high-bias peak arises from tunneling into subband 2. As the energy splitting $E_{2}-E_{1}$ exceeds the LO phonon energy, inelastic intersubband scattering is very efficient. In order to check the relevance of such processes, we performed photoluminescence measurements. Even when the electrons were injected into subband 2, we found only recombination from subband 1 , in agreement with previous work [15]. This demonstrates that the relaxation rate $\Gamma_{\text {rel }}^{21}$ exceeds the tunneling rates (in particular $\Gamma_{C}^{2}$ ). Thus, the high-bias peak results from an inelastic sequential process consisting of emitter-barrier tunneling into subband 2 , relaxation to subband 1 , and collector-barrier tunneling.

Figure 2(b) displays the $I(V)$ curve of a device with $d_{p}=250 \mathrm{~nm}$ pillar diameter. It shows less-pronounced peaks compared to the large-area sample (only the rising edge of the second peak is visible). The submicron device exhibits, in addition to the vertical quantization, a lateral confinement due to mid-band-gap Fermi-level pinning at the pillar sidewalls. Thus, two disk-shaped quantum dots
$A$ and $B$ are formed [see Fig. 1(a)]. Note that the current peaks are slightly shifted to higher bias voltage compared to the large-area sample as a consequence of the lateral confinement. The electronic diameter $d_{e}$ of the dots is much smaller than the pillar diameter due to a depletion layer at the pillar surface. By comparing the peak currents of the large-area sample and the submicron device, we estimate $d_{e} \approx 70$ and $85 \mathrm{~nm}$ at the low- and high-bias peaks, respectively. The most striking feature in Fig. 2(b) is the staircaselike fine structure superimposed on the rising edge of both current peaks. Similar staircases were previously observed as a consequence of SET through discrete levels in single quantum dots [2-4]. In contrast, sequential SET through two quantum dots in series was shown to lead to sharp peaks in the $I(V)$ curve [6-10]. Hence, we rule out that the staircases in Fig. 2(b) arise from elastic sequential tunneling but interpret our data in terms of SET through discrete states extended over both dots due to coherent interdot coupling. Note moreover that we attribute oscillatory features on the plateaus of the current steps to density-of-states fluctuations in the contacts [16].

The single-particle states of the DDS are the eigenfunctions of the Hamiltonian $H=\mathbf{p}^{2} / 2 m^{*}+V(\mathbf{r})$, where $m^{*}$ is the effective electron mass. The problem separates into $H=H_{z}+H_{\|}$with $H_{z}=p_{z}^{2} / 2 m^{*}+$ $V_{z}(z)$ and $H_{\|}=\mathbf{p}_{\|}^{2} / 2 m^{*}+V_{\|}\left(\mathbf{r}_{\|}\right)$, since the confinement potential of the DDS splits into $V(\mathbf{r})=V_{z}(z)+V_{\|}\left(\mathbf{r}_{\|}\right)$. The eigenvalues of $H_{z}$ coincide with the subband edges $E_{1}$ and $E_{2}$ of the laterally unconfined case. Denoting the eigenvalues of $H_{\|}$with $E_{n, l}$, we obtain two ladders of discrete single-particle levels $E_{1}+E_{n, l}$ and $E_{2}+E_{n, l}$ as exact eigenvalues $E_{1 / 2, n, l}$ of $H$.

For zero bias the DDS is empty, because all discrete levels lie above the chemical potential $\mu_{E}^{\mathrm{ch}}$ in the emitter [Fig. 1(b), top]. The current is basically zero. By applying a bias voltage $V$, the DDS levels are reduced in energy with respect to the electrochemical potential $\mu_{E}$ of the emitter [Fig. 1(b), bottom]. If the accumulation of more than one electron in the DDS at a time is irrelevant, current steps with magnitude

$$
\Delta I=2 e \Gamma_{E} \Gamma_{C} /\left(2 \Gamma_{E}+\Gamma_{C}\right)
$$

arise from consecutive tunneling of single electrons through the device [17], whenever a single-particle level $E_{1 / 2, n, l}$ falls below the electrochemical potential of the emitter. The positions of these current steps

$$
V_{1 / 2, n, l}=\left(E_{1 / 2, n, l}-\mu_{E}^{\mathrm{ch}}\right) / e \alpha_{1 / 2}
$$

provide direct experimental access to the single-particle levels of the DDS $\left(\alpha_{1 / 2}\right.$ denote the voltage-to-energy conversion coefficients to $E_{1 / 2}$ ) [18].

In our experiment we observe two separate $I(V)$ staircases. As the low-bias peak in the $I(V)$ curve of the largearea device arises from tunneling through subband 1 , we 
attribute the low-bias staircase to SET through the corresponding DDS levels $E_{1, n, l}$. Charging of the DDS with more than one electron at a time is extremely improbable in this regime, since tunneling electrons can much easier leave than enter the DDS $\left(\Gamma_{E}^{1} \ll \Gamma_{C}^{1}\right)$. Therefore, only single-particle states contribute to SET. In the regime between the two staircases the current decreases slightly. This indicates (i) that low-energy DDS levels are lost for SET by being pulled below the conduction-band edge in the emitter and (ii) that the coupling strength between the emitter and high-energy DDS levels $E_{1, n, l}$ is reduced. The current rises again at the threshold of the high-bias staircase which we relate to SET into the DDS levels $E_{2, n, l}$, since the high-bias peak in the $I(V)$ curve of the largearea device results from tunneling into subband 2 . If the relaxation rate $\Gamma_{\text {rel }}^{21}$ from $E_{2, n, l}$ to $E_{1, n^{\prime}, l^{\prime}}$ exceeds the tunneling rates, the amount of charge accumulation is determined by $\Gamma_{E}^{2}$ and $\Gamma_{C}^{1}$. As the height of the collector barrier is reduced by the bias voltage compared to the emitter barrier, charging is improbable $\left(\Gamma_{E}^{2}<\Gamma_{C}^{1}\right)$. In contrast, strong charging is the consequence when the relaxation is negligible, since electrons can much easier enter than leave the DDS $\left(\Gamma_{E}^{2} \gg \Gamma_{C}^{2}\right)$. In this case, the consideration of single-particle levels is not sufficient but many-electron states have to be taken into account.

Therefore, the key question is whether the relaxation in the DDS is faster than tunneling as observed in large-area devices. To answer this question, we consider the magnitude of the current steps: The step height $\Delta I_{2} \approx 200 \mathrm{pA}$ of the high-bias $I(V)$ staircase exceeds the value $\Delta I_{1} \approx$ $4 \mathrm{pA}$ of the low-bias staircase by 2 orders of magnitude [comparable to the height ratio of the current peaks in the $I(V)$ curve of the large-area device]. According to Eq. (1) the smaller one of the two tunneling rates limits the step height. Thus, $\Delta I_{2}$ would be limited by $\Gamma_{C}^{2}$ if there were no relaxation in the DDS, while $\Delta I_{1}$ is limited by $\Gamma_{E}^{1}$. Within this picture, the step heights of both staircases should be of the same order of magnitude in contrast to our data. If, however, the relaxation is faster than tunneling, $\Delta I_{2}$ is determined by $\Gamma_{E}^{2}\left(\gg \Gamma_{E}^{1}\right)$ and therefore much larger than $\Delta I_{1}$, in agreement with experiment. Hence, we conclude that no charging occurs, i.e., the high-bias staircase corresponds to the single-particle states $E_{2, n, l}$ of the DDS.

In order to support our model, we applied a magnetic field $B \| I$. The field increases the lateral confinement shifting the ground-state energy $E_{0,0}$ of $H_{\|}$to higher values. Therefore, the first current steps of the two $I(V)$ staircases which we relate to $E_{1,0,0}$ and $E_{2,0,0}$ shift in Figs. 3(a) and 3(b) to higher bias voltage. To eliminate the magnetic-field dependence of the emitter states [4], we take the position of the first step as reference and plot in Fig. 4(a) the differences $V_{1, n, l}-V_{1,0,0}$ of the low-bias staircase. They move monotonously to lower bias/energy at high magnetic fields. Such a field dependence is expected for the single-particle levels of a disk-shaped quantum dot [19]. Figure 4(b) shows the corresponding differences $V_{2, n, l}-V_{2,0,0}$ of the high-bias

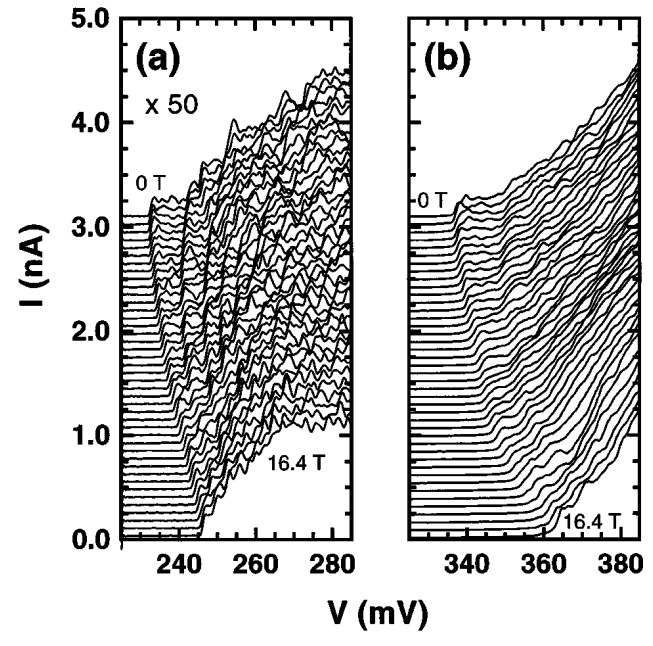

FIG. 3. Magnetic-field dependence of the low- and high-bias $I(V)$ staircases [(a) and (b)] of the submicron device $(B \| I)$. Curves are taken in steps of $0.4 \mathrm{~T}$ between $B=0$ and $16.4 \mathrm{~T}$ and vertically offset for clarity.

staircase. Their magnetic-field dependence is qualitatively in good agreement with the data of the lowbias staircase [e.g., compare the two lowest curves in Figs. 4(a) and 4(b)]. This confirms the absence of charging. Thus, the low- and high-bias staircases reflect the single-particle levels $E_{1, n, l}$ and $E_{2, n, l}$ of the DDS.

For a quantitative analysis, we calculate the biasvoltage differences of Fig. 4 according to Eq. (2),

$$
\left(V_{1 / 2, n, l}-V_{1 / 2,0,0}\right)=\left(E_{n, l}-E_{0,0}\right) / e \alpha_{1 / 2} .
$$

Assuming the potential $V_{\|}\left(\mathbf{r}_{\|}\right)=\frac{1}{2} m^{*} \omega_{0}^{2} r_{\|}^{2}$ of a $2 \mathrm{D}$ harmonic oscillator for lateral confinement, we have

$$
E_{n, l}=(2 n+1+|l|) \sqrt{\left(\hbar \omega_{0}\right)^{2}+\left(\hbar \omega_{c} / 2\right)^{2}}+(l / 2) \hbar \omega_{c}
$$

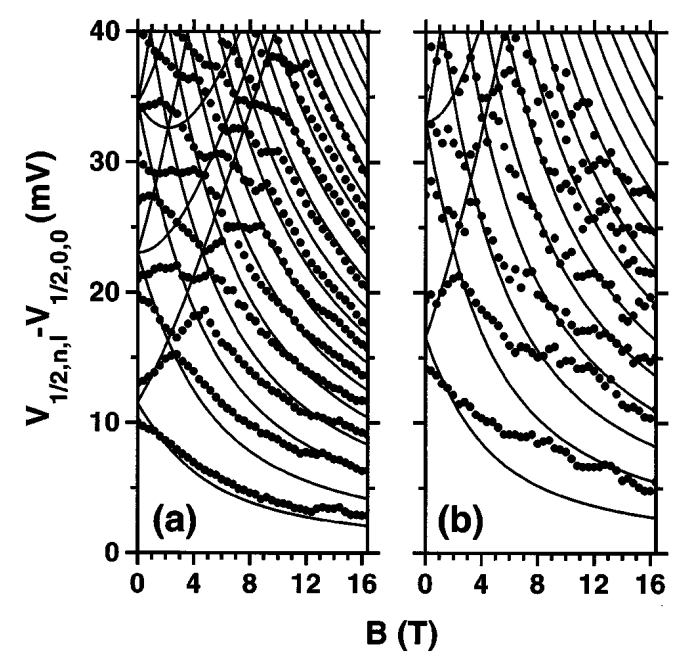

FIG. 4. Magnetic-field dependence of the current-step positions (solid circles) of the low- and high-bias $I(V)$ staircases [(a) and (b)]. The step positions are plotted with respect to the position of the first step and compared with the single-particle spectrum of a 2D harmonic oscillator (solid lines). 
for the eigenvalues of $H_{\|}$in a magnetic field $B \| I$ with $\omega_{c}=e B / m^{*}$ [19]. First, we consider the data of the low-bias staircase in Fig. 4(a) and determine $\hbar \omega_{0}$ from the zero-field values of the two lowest differences as $\hbar \omega_{0}=e \alpha_{1}\left(V_{1,0, \pm 1}-V_{1,0,0}\right)$. Estimating $\alpha_{1}=0.46$ from the Poisson solver for the levels $E_{1, n, l}$, we obtain $\hbar \omega_{0}=5.3 \mathrm{meV}$. With this value, we calculate the complete set $V_{1, n, l}-V_{1,0,0}$ from Eqs. (3) and (4). The agreement between theory and experiment in Fig. 4(a) is fairly good. Close to degeneracies in the theoretical data, however, intricate anticrossings are observed experimentally. This indicates that the rotational symmetry of our DDS is not perfect. The lateral extension of the ground state can be estimated as the distance $d_{c}=2 \sqrt{2 \hbar / m^{*} \omega_{0}}$ between the classical oscillator-turning points at energy $E_{0,0}$. Our result of $d_{c} \approx 40 \mathrm{~nm}$ is smaller than the electronic diameter $d_{e} \approx 70 \mathrm{~nm}$ at the low-bias current peak, where also excited states with a larger lateral extension contribute to SET (states of zero-field energy $M \hbar \omega_{0}$ have a classical diameter of $\sqrt{M} d_{c}$ ). From the data of the high-bias staircase in Fig. 4(b), we determine $\hbar \omega_{0}$ according to the procedure described above for the low-bias staircase. Calculating $\alpha_{2}=0.29$ for $E_{2, n, l}$, we obtain $\hbar \omega_{0}=4.8 \mathrm{meV}$. This value is strikingly close to that determined for $E_{1, n, l}$, which illustrates the homogeneity of the lateral confinement of our DDS.

Now, we discuss the efficient relaxation from the DDS levels $E_{2, n, l}$ to $E_{1, n^{\prime}, l^{\prime}}$, for which we collected strong evidence from both the heights and magneticfield dependence of the current steps. As the relaxation rate $\Gamma_{\text {rel }}^{21}$ exceeds the tunneling rates $\Gamma_{E}^{1} \sim 10^{7} \mathrm{~s}^{-1}$ and $\Gamma_{E}^{2} \sim 10^{9} \mathrm{~s}^{-1}$ [estimated from $\Delta I_{1}$ and $\Delta I_{2}$ according to Eq. (1)], the relaxation occurs on a ns time scale or faster. In contrast to large-area devices, LO phonon processes cannot account for the relaxation in the DDS, as they are energetically limited to very specific values of the applied bias voltage. Moreover, LA and LO + LA phonon scattering were predicted to become inefficient in small quantum dots at high LA phonon energies [20]. Our SET experiment gives no evidence for this "phonon-relaxation bottleneck." Note, however, that other relaxation mechanisms such as Coulomb scattering with free electrons in the contacts may be relevant as well [21].

In summary, we investigated single-electron transport through a vertically coupled double-dot system. Two series of current steps with different heights were observed in the $I(V)$ curve. Their magnetic-field dependence agrees with that of the single-particle levels of a 2D harmonic oscillator. Our results support the forma- tion of discrete states extended over both identical dots due to coherent interdot coupling. Thus, the DDS represents an ionized artificial hydrogen molecule which is detuned by the applied bias voltage. From the absence of many-particle phenomena, we conclude that the energyrelaxation rate in the DDS exceeds $10^{9} \mathrm{~s}^{-1}$.

We thank B. Schönherr for reactive-ion etching, D. Bertram for optical measurements, and U. Bockelmann, B. Farid, J.H. Oh, and J. Weis for discussions. This work was funded by the Bundesministerium für Bildung, Wissenschaft, Forschung und Technologie.

[1] For recent reviews, see U. Meirav and E. B. Foxman, Semicond. Sci. Technol. 10, 255 (1995); R. C. Ashoori, Nature (London) 379, 413 (1996).

[2] M. Tewordt et al., J. Phys. Condens. Matter 2, 8969 (1990).

[3] B. Su et al., Science 255, 313 (1992).

[4] T. Schmidt et al., Phys. Rev. B 51, 5570 (1995).

[5] M. A. Reed et al., Adv. Solid State Phys. 29, 267 (1989).

[6] M. Tewordt et al., Appl. Phys. Lett. 60, 595 (1992); Phys. Rev. B 49, 8071 (1994).

[7] U. Sivan et al., Europhys. Lett. 25, 605 (1994).

[8] M. Kemerink and L. W. Molenkamp, Appl. Phys. Lett. 65, 1012 (1994); L. W. Molenkamp et al., Phys. Rev. Lett. 75, 4282 (1995).

[9] F. Hofmann et al., Phys. Rev. B 51, 13872 (1995).

[10] N.C. van der Vaart et al., Phys. Rev. Lett. 74, 4702 (1995).

[11] F. R. Waugh et al., Phys. Rev. Lett. 75, 705 (1995).

[12] R. H. Blick et al., Phys. Rev. B 53, 7899 (1996).

[13] D. Dixon et al., Phys. Rev. B 53, 12625 (1996).

[14] I. M. Ruzin et al., Phys. Rev. B 45, 13469 (1992); G. W. Bryant, Phys. Rev. B 48, 8024 (1993); G. Klimeck et al., Phys. Rev. B 50, 2316 (1994); J. J. Palacios and P. Hawrylak, Phys. Rev. B 51, 1769 (1995); J. M. Golden and B. I. Halperin, Phys. Rev. B 53, 3893 (1996); H. Imamura et al., ibid. 53, 12613 (1996); J. H. Oh et al., ibid. 53, 13264 (1996).

[15] T. S. Turner et al., Solid State Electron. 37, 721 (1994).

[16] T. Schmidt et al,, Europhys. Lett. 36, 61 (1996).

[17] L. Y. Chen and C. S. Ting, Phys. Rev. B 44, 5916 (1991).

[18] The charging energy to put the first electron on the DDS is omitted in Eq. (2) as it is similar for all $E_{1 / 2, n, l}$.

[19] V. Fock, Z. Phys. 47, 446 (1928).

[20] U. Bockelmann and G. Bastard, Phys. Rev. B 42, 8947 (1990); T. Inoshita and H. Sakaki, Phys. Rev. B 46, 7260 (1992); H. Benisty, Phys. Rev. B 51, 13281 (1995).

[21] U. Bockelmann and T. Egeler, Phys. Rev. B 46, 15574 (1992). 